\title{
Locating waste pipelines to minimize their impact on marine environment
}

\author{
Teresa Cáceres ${ }^{a}$, Juan A. Mesa ${ }^{b}$, Francisco A. Ortega ${ }^{\mathrm{c}}$
}

aepartment of Applied Mathematics I, Computer Engineering Higher Technical School, University of Seville, Spain

b Department of Applied Mathematics II, Engineering Higher Technical School, University of Seville, Spain

Department of Applied Mathematics I, Architecture Higher Technical School, University of Seville, Spain

\begin{abstract}
A waste pipeline, considered as an undesirable facility, is to be located in a coastal region. Two criteria are taken into account, the Euclidean distance from a given set of protected areas (coral reefs and sandbanks) and a utility function related to the pipe length, both to be maximized. The paper describes a methodology to obtain an efficient set of points where the extreme of a marine pipeline should be located. Since the formulation of the model is based on the zone Voronoi diagram, the computational complexity of the solving procedure is low.
\end{abstract}

Keywords: Undesirable facility location; Forbidden regions; Zone Voronoi diagram

\section{Introduction}

The coastal fringe is the territory where marine, air and terrestrial environments interrelate. Here very diverse and fragile ecosystems coexist, although subjected in many cases to increasing degradation due to industrial and urban developments disrespectful to the environment.

Nearly two-thirds of the world's population live along the seaboard. In Spain this proportion can be estimated as $69 \%$ by using data corresponding to year 2001 (Instituto Nacional de Estadística, 2004). Almost a quarter of the Mediterranean littoral in Spain is artificial surface. Most marine contamination is produced on land. Each year, 10 billion tons of industrial and urban sewage are directly poured into the Mediterranean sea, and $90 \%$ is untreated. Pollution specifically affects oceanic prairies and coral reefs due to decreasing vegetable biomass and biological diversity (see Worldwide Fund for Nature/Adena, 2000).

Posidonia oceanica (Linnaeus) Delile is a plant with leaves, flowers and fruit, similar to those plants which live in forests and gardens, but which lives in the sea between the surface and a depth of $50 \mathrm{~m}$, where there is still enough light for photosynthesis. It is endemic to the Mediterranean sea and, by providing the principal 
source of oxygenation of the Mediterranean sea, it is its most important ecosystem. The Posidonia meadow produces a barrier reef which maintains the balance of the littoral sedimentation since their long leaves restrain the swell thereby protecting the littoral from erosion by minimizing the impact of the waves on the beaches (see Marbá et al., 1996). The Posidonia meadow is the habitat of more than 400 plant species and 1000 animal species; it provides shelter, food and an adequate environment for the reproduction of many profitable species. Due to its ecological role, this sea-grass is a protected species in Spain and in France.

Nowadays, Posidonia oceanica is decreasing on the coastal fringe due to several factors:

1. Sea contamination, fundamentally from terrestrial sources, produces a subsequent reduction in water quality, which obstructs photosynthesis, thereby causing the death of the plant.

2. Illegal coastal drag fishing is harmful because of its strong physical impact.

3. Public works on the coastal fringe (sports harbours, breakwaters, regeneration of beaches) modify the littoral dynamic and therefore the environmental conditions of the sea floor.

4. The frequent seasonal anchorage of ships in the same places on the coast can damage the sea floor.

The European Union (EU) recognizes the need to protect the habitats and marine ecosystems in coastal waters; in particular, meadows of Posidonia oceanica, coral reefs and sandbanks (see EU Directive, 1992). Following the corresponding EU-directives, government agencies are at present promoting the planning and the management of coastal environments.

Research carried out in different zones, to determine the distribution and boundaries of the different substratum and sediments of sea-grass beds along the coast, commonly generate a database of visual, topographic and thematic maps so that the analysis by means of a Geographic Information System (GIS) can be performed (see Calzadilla Pérez et al., 2002; Jayatissa et al., 2002; Melloul and Collin, 2002).

Once the geographical location of the study area has been established, vigilance and control systems of these habitats are applied in order to detect, by aerial photographs, turbidities of the water column's vertical structure. For this purpose, the collected satellite images are processed with the GIS in order to assess the interaction between the detected disturbances and the Posidonia oceanica beds. In Fig. 1 a satellite image is shown of the south-west coast of Spain near the mouth of the Tinto river (province of Huelva, Andalusia).

Simulation tools, which have been recently developed to assess the water quality, integrate the software to manage teledetection and geo-statistical information. The images generated by those systems are typically planar (see He, 2003). Therefore, the formulation of problems on a plane makes sense although their nature is tridimensional.

The setting considered in this paper is a geometrical abstraction of a coastal scenario which consists of a planar region, which includes zones of biological interest, whose left-hand side represents the littoral line. Taking into account the existence of upper and lower boundaries for the township in charge of the installation of the pipeline, the scenario will be assumed to be a rectangular strip. Here one waste pipeline (as Fig. 2 shows) must be located perpendicular to the coastal line so that its emissions have the lowest impact on marine environment.

The modern plastic materials, such as polyethylene, used for the fabrication of outfall pipelines, are essentially immune to the corrosive effects of seawater and to the attack by marine organisms. Moreover, due to the

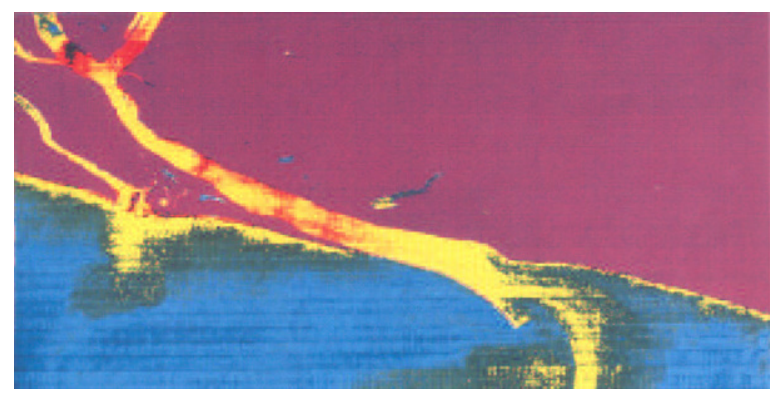

Fig. 1. Teledetection by satellite. 


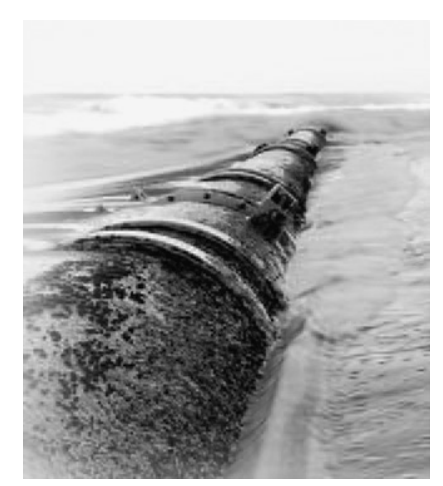

Fig. 2. Marine pipe.

flexibility of this material, the route of an outfall pipeline can easily avoid obstacles, hazards and environmentally sensitive areas. In planning a marine outfall the first step should be to determine a suitable location for the diffuser. The determination of the location and design of the diffuser should be based on obtaining adequate distance from sensitive areas and sufficient depth, dispersion and/or die-off of pollutants commensurate with the level of treatment prior to discharge to assure negligible environmental or health impact (Reiff, 2002).

The total cost of an outfall is dependent, to a great degree, on the length and diameter of the outfall pipeline. The length can be determined by the location of environmentally sensitive areas, beaches and water sport areas, the slope of the ocean floor, the prevailing wind direction and the direction and velocity of currents. In this paper, the length $(x \geqslant 0)$ of the outfall pipeline is associated to a utility function $\operatorname{Util}(x) \geqslant 0$ whose outline satisfies the following properties:

1. The pipeline length must be strictly positive (Util( 0$)=0$; Util $(x)>0, \forall x>0$ ).

2. Function Util $(x)$ is strictly increasing for $x>0$ up to a threshold value $x^{*}>0$ where a maximum is attained. Within reason, the further the outfall is from the coast, the better.

3. Function Util $(x)$ asymptotically decreases to a zero level since the excessive length puts up the cost of installation.

A generalized gamma function

$$
\operatorname{Util}(x)=x^{\alpha} \mathrm{e}^{-\beta x}, \quad \forall x \geqslant 0,
$$

commonly used to develop the friction factors in the models of trip distribution (Ortúzar and Willumsen, 2001), satisfies the previous spatial behaviour (Fig. 3).

Parameters $\alpha$ and $\beta$ are positive and have yet to be calibrated. The procedure of parameter calibration must be based on the statistical analysis of distribution of observed lengths of waste pipelines. In this sense, the

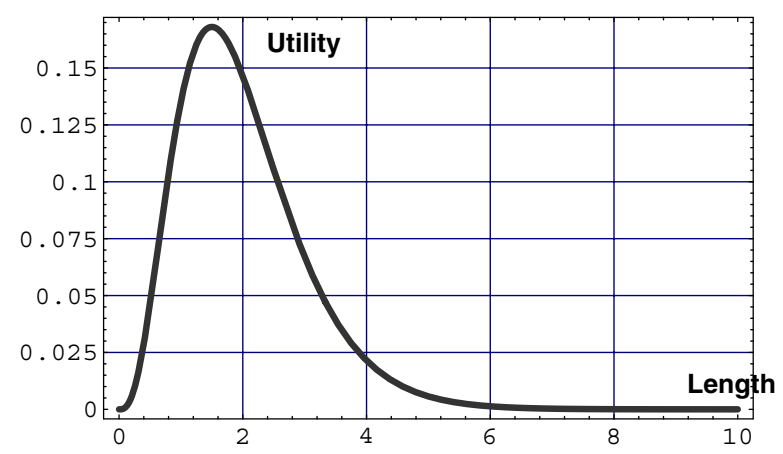

Fig. 3. An instance of the generalized gamma density function. 
method of least-squares can be used to determine the best fit parameters $\alpha$ and $\beta$ for the pipeline lengths collected in a database.

Although these parameters appear non-linearly in the definition of the utility function, a transformation can be applied to obtain an appropriate linear combination in the unknown parameters. In particular, taking logarithms in the expression $y=x^{\alpha} \mathrm{e}^{-\beta x}$

$$
\log y=\alpha \log x-\beta x \Rightarrow \frac{\log y}{\log x}=\alpha-\beta \frac{x}{\log x} .
$$

Therefore, given a list of statistical data $\left(x_{d}, y_{d}\right), d \in D$, where $x_{d}$ indicates the length of the outfall pipeline whose utility is estimated by $y_{d}$, the optimal values for the variables $\alpha$ and $\beta$ in the least-squares sense can be calculated by means of the expressions:

$$
\begin{aligned}
& \beta=-\frac{|D|\left(\sum \frac{x_{d}}{\log x_{d}} \frac{\log y_{d}}{\log x_{d}}\right)-\left(\sum \frac{x_{d}}{\log x_{d}}\right)\left(\sum \frac{\log y_{d}}{\log x_{d}}\right)}{|D| \sum\left(\frac{x_{d}}{\log x_{d}}\right)^{2}-\left(\sum \frac{x_{d}}{\log x_{d}}\right)^{2}} ; \\
& \alpha=\frac{\left(\sum \frac{\log y_{d}}{\log x_{d}}\right)\left(\sum\left(\frac{x_{d}}{\log x_{d}}\right)^{2}\right)-\left(\sum \frac{x_{d}}{\log x_{d}}\right)\left(\sum \frac{x_{d}}{\log x_{d}} \frac{\log y_{d}}{\log x_{d}}\right)}{|D| \sum\left(\frac{x_{d}}{\log x_{d}}\right)^{2}-\left(\sum \frac{x_{d}}{\log x_{d}}\right)^{2}} .
\end{aligned}
$$

The aim of this article is to analyse the problem of locating a waste pipeline on a coastal region if forbidden regions exist in order that both the impact on marine habitats is minimized and a utility function associated to pipe length is maximized. The paper is organized as follows: Section 2 describes the methodology of solving the maxmin location problem. Section 3 obtains the optimum for the utility function. Section 4 states the model of location in the plane in formal terms as a bi-criterion problem, showing an example. In Section 5 , conclusions are presented and several extensions of this problem are introduced.

\section{The maxmin location problem}

\subsection{Formulation}

Let $X(x, y)$ be the end of a waste pipeline parallel to axis $O X$, inside a bounded rectangle in the first square of the Cartesian plane, whose lower left-hand vertex coincides with the origin of the coordinates.

We assume for the sake of simplicity that the areas to be protected can be enclosed in the interior of circles (coral reefs) and rectangles parallel to Cartesian axes (sandbanks and meadows of Posidonia oceanica). There are two technical reasons for assuming these shapes for the sensitive regions: first, the underlying distance in the scenario is Euclidean, and subsequently the level curves of iso-affectation will be circumferences in the absence of predominant currents, and secondly, the digitization required to process the images obtained by spatial sampling in the coastal zone is based on the previous existence of a rectangular grid which supports maps of colour variations (see Caerio et al., 2003).

In general coral reefs have irregular shapes (usually non-convex, close to a fractal shape) and the orientation of sandbank boundaries is dependent on the prevailing direction of the waves. Nevertheless, the possibility of overlapping those basic figures (circles and rectangles) of a small size provides a reasonable level of accuracy in the assumption. Evidently, a higher resolution in the maps will lead to a greater computational effort for the determination of solutions.

When considering a medium scale, a polygonal approximation to the morphology of the protected habitats can be appropriate. Notice that only one homogeneous type of polygonal contour is assumed in the paper: forbidden regions are iso-orientated rectangles. This assumption provides an immediate decomposition of the study region into cells, which are also rectangular, where the curves containing efficient points can be regularly determined (see figure which illustrates the last case considered in the Appendix). The methodology developed for this context can be easily adapted to solve the corresponding location problem in presence of polygonal regions which are non-necessarily rectangular. 
Let $\Omega=[0, M] \times[0, L]$ be a subset of $\mathbb{R}^{2}$, with $M, L>0$, which denotes the region under consideration. Let $\mathscr{C}=\left\{C_{i}: i \in I\right\}$ be a set of forbidden circular regions, with respective centres at $A_{i}\left(a_{i 1}, a_{i 2}\right)$ and non-negative radii $r_{i}$, which represents the set of coral reefs which need protection in relation to pollutant emissions. In addition, let $\mathscr{R}=\left\{R_{j}: j \in J\right\}$ be a set of rectangular regions, with the respective lower left-hand vertices denoted by $V_{j}\left(v_{j 1}, v_{j 2}\right)$, length $l_{j}$ and height $h_{j}$, which are assumed to be the Posidonia oceanica prairies or sandbanks (rectangular bands parallel to coastal fringe) considered as zones of biological interest. These circles and rectangles are completely or partially contained in $\Omega$.

In general, let $\mathscr{Z}=\left\{Z_{k}: k \in K\right\}$ represent a set of circular or rectangular zones. The feasible region for the problem is $\mathscr{S}=\Omega \backslash\left(\cup_{k} Z_{k}\right)$. Since each $Z_{k}$ is a simply-connected closed set, the distance from a point $P$ to a zone $Z_{k}$ can be defined as

$$
d\left(P, Z_{k}\right)=\min _{Q \in Z_{k}} d_{2}(P, Q),
$$

where $d_{2}(\cdot, \cdot)$ denotes the Euclidean distance.

Once the intensity of the waste dispersion decreases with the Euclidean distance from the diffuser $X$, the natural objective will coincide with the formulation of the corresponding 1-Maxmin problem; namely,

$$
\max _{X \in \mathscr{S}} \operatorname{Dmin}(X) \equiv \min _{k}\left\{d\left(X, Z_{k}\right)\right\} .
$$

For a comprehensive survey of the literature on inverse optimization, the reader is referred to Qin et al. (2003).

\subsection{A concise review}

This maxmin problem can be transformed into an equivalent formulation:

$$
\begin{aligned}
& \max \delta \\
& \text { s.t. } \\
& \delta \leqslant d\left(X, Z_{k}\right), \quad k \in K \\
& X \in \mathscr{S},
\end{aligned} \rightarrow\left\{\begin{array}{l}
\max \delta \\
\text { s.t. } \\
\delta \leqslant \min _{Q \in R_{j}} d_{2}(X, Q), \quad j \in J, \\
\delta \leqslant \min _{Q \in C_{i}} d_{2}(X, Q), \quad i \in I, \\
X \in \mathscr{S}
\end{array}\right.
$$

Analogous formulations for the aforementioned problem can be found in the literature designed for the modelling of real scenarios. For instance,

1. Melachrinoudis and Cullinane (1985) used this maxmim objective to locate one undesirable facility within a geographical region; in particular, they applied the model to the state of Massachusetts, approximating the forbidden regions (50 existing cities) by means of circles. Karush-Kuhn-Tucker conditions were used to identify the set of local maxima.

2. Fernandez et al. (1997) used two approaches: the first methodology was based on the Karush-KuhnTucker conditions and the second geometrical development exploited the concept of proximity of segment, to solve the problem:

$$
\left\{\begin{array}{l}
\max \delta \\
\text { s.t. } \\
\delta \leqslant \min _{Q \in S_{j}} d_{2}(X, Q), \quad j \in J, \\
X \in \mathscr{S},
\end{array}\right.
$$

where each $S_{j}$ was a forbidden polygonal region. The authors applied the methodology to locate an incineration plant in a region of the South of Spain where the predominant wind directions indicate a specific shape for the protected area centred at each population centre.

3. Tuy et al. (2003, in press) proposed an efficient difference-of-convex (DC) optimization algorithm to solve the maxmin problem 


$$
\left\{\begin{array}{l}
\max \delta \\
\text { s.t. } \\
\delta \leqslant \min _{Q \in D_{i}} d_{2}(X, Q), \quad i \in I, \\
X \in \mathscr{S},
\end{array}\right.
$$

where each $D_{i}$ was a circular region to be preserved.

Two examples were presented in this last paper to illustrate the methodology followed. One of them involved 11 circles with different centres and radii, where 45 iterations were necessary so that the DC algorithm would reach the optimal solution. Fig. 4 shows the largest empty circle from the set of existing circles considered in a rectangular region.

\subsection{Our methodological proposal}

Although different non-convex quadratic programming methods can be applied to solve this largest empty ball problem, we propose an approach based on the concept of the area Voronoi diagram (Okabe et al., 2000) to find a finite dominating set of circle centres from which global optima can be determined.

The area Voronoi diagram can be constructed from its corresponding line Voronoi diagram. Let $\mathscr{E}=\left(\bigcup_{k \in K} \partial Z_{k}\right) \cup \partial \Omega$ represent the union of the set of boundaries of $\mathscr{Z}$ and the four edges of rectangle $\Omega$. In our model the lines which delimit each forbidden zone $\partial Z_{k}$ belong to the following types:

1. Scenario boundaries; namely, axis $O X(y=0)$, axis $O Y(x=0)$ and delimitations $x=M, y=L$.

2. Contour of circle $C(A, r)$ centred at $A\left(a_{1}, a_{2}\right)$ and with radius $r>0$.

3. Contour of rectangle $R(V, l, h)$, whose lower left-hand vertex is located at point $V\left(v_{1}, v_{2}\right)$ and whose horizontal and vertical dimensions are respectively $l>0$ and $h>0$.

Let $\left|\partial Z_{k}\right|$ be the number of line segments and circumference arcs which enclose protected area $Z_{k}$. Define $|\mathscr{E}|$ as the total number of lines to be considered for building the line Voronoi diagram: $|\mathscr{E}|=\sum_{k \in K}\left|\partial Z_{k}\right|$. The following proposition deals with the computation of the bisector curves for all possible pairs of boundary types of the aforementioned zones.

Proposition 1. Bisector curves for zone pairs are piece-wise curves composed of straight lines, parabolas andlor hyperbolas.

Therefore, the edges of this line Voronoi diagram are always simple continuous curves. An application of Caratheodory's representation theorem on the plane (see Rockafellar, 1970) leads us to state that the diagram vertices may be defined as those points shared by three or more Voronoi edges. Due to this fact, the area Voronoi diagram can be efficiently generated in time $\mathrm{O}(|\mathscr{E}| \log |\mathscr{E}|)$ following the procedure suggested by Okabe

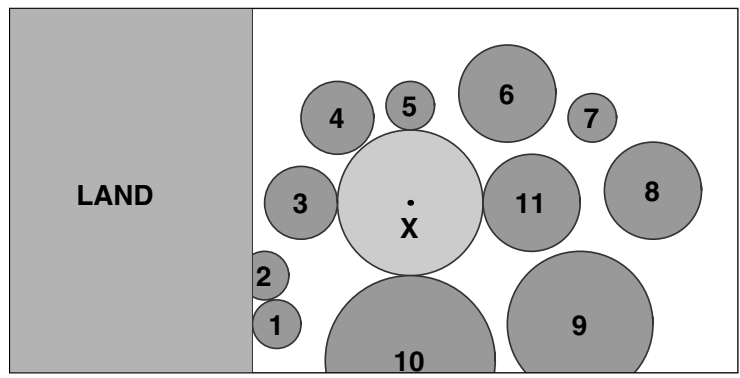

Fig. 4. Largest empty circle. 


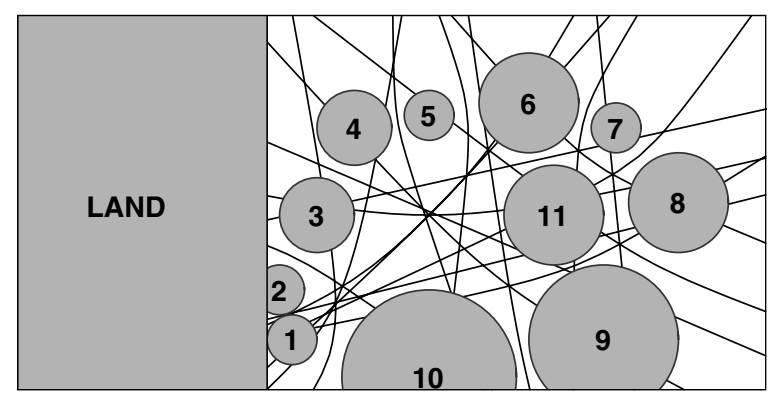

Fig. 5. The line Voronoi diagram.

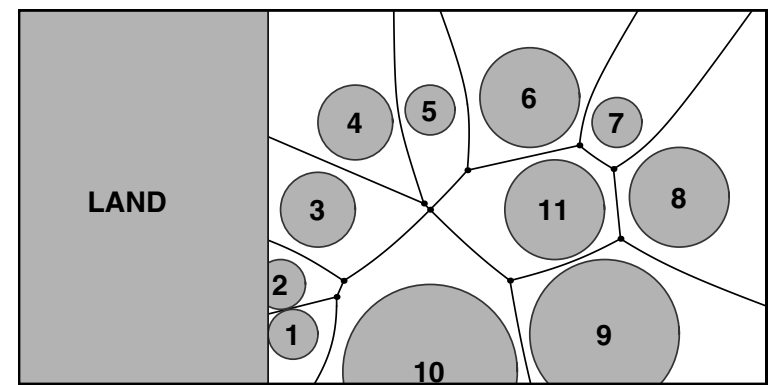

Fig. 6. The area Voronoi diagram.

et al. (2000); namely, first build the line area Voronoi diagram associated to edges which represent the boundaries of forbidden zones, and then delete the superfluous edges. The aforementioned computational complexity is ensured by the property labelled $O K A 1$ in that book (page 187).

Figs. 5 and 6 respectively show the line Voronoi diagram and the area Voronoi diagram associated with the example of Tuy's paper.

Once the line Voronoi diagram has been built, the largest empty circle problem:

Given a set of distinct rectangular and circular zones $\mathscr{Z}$ find the largest empty circle whose centre is in set $\Omega$ can be solved.

\section{Maximizing the utility function}

The two parameters $\alpha$ and $\beta$ of function

$$
y=\operatorname{Util}(x)=x^{\alpha} \mathrm{e}^{-\beta x}
$$

allow the analyst to fine-tune the utility associated with the pipeline length. Its calibration can be based on the coincidence between statistical parameters of pipeline samples (for an identical purpose) and theoretical values of the utility function where relevant properties exist. Among these are:

1. Since function $y=x^{\alpha} \mathrm{e}^{-\beta x}$, is a differentiable function, the study of the sign of its derivative function

$$
y^{\prime}=(\alpha-\beta x) x^{\alpha-1} \mathrm{e}^{-\beta x},
$$

in the interval $x \in(0, M)$, indicates that its only maximum is attained at point $x^{*}=\frac{\alpha}{\beta}$ for $\alpha>1$. A typical length for the pipeline length average is $\bar{l}=200 \mathrm{~m}$ (Reiff, 2002). Therefore, the assumption $\frac{\alpha}{\beta}=\bar{l}$ permits a first reduction of the number of parameters to calibrate. 
2. Taking into account that

$$
y^{\prime}=\frac{\alpha-\beta x}{x} y
$$

a new derivation can be performed, yielding

$$
y^{\prime \prime}=\frac{(\alpha-\beta x)^{2}-\alpha}{x^{2}} y .
$$

Since $y>0$, two inflexion points can easily be deduced by imposing condition $y^{\prime \prime}=0$ :

$$
x_{1}=\frac{\alpha}{\beta}-\frac{\sqrt{\alpha}}{\beta} ; \quad x_{2}=\frac{\alpha}{\beta}+\frac{\sqrt{\alpha}}{\beta} .
$$

The second value indicates that the asymptotical decrease to zero for the utility function starts when the pipeline length exceeds a distance $\frac{\sqrt{\alpha}}{\beta}$ from the length average $\bar{l}=\frac{\alpha}{\beta}$. Together with the aforementioned property, this new property can also be used to eventually determine the parameter values.

3. Assuming an approach for a single-parameter $\beta$ which can take value 1 , hence, $\alpha$ is the only parameter which characterizes the maximum of the utility function. Moreover, if we are interested in a representation of the component of utility in terms of probability, a simple factor is all that is required to normalize the utility function; namely,

$$
\operatorname{Util}^{*}(x)=\frac{1}{\Gamma(\alpha+1)} x^{\alpha} \mathrm{e}^{-x}, \quad \alpha>0 .
$$

The normalization factor is based on the Euler gamma function:

$$
\Gamma(z)=\int_{0}^{\infty} t^{z-1} \mathrm{e}^{-t} \mathrm{~d} t, \quad z>0 .
$$

The general properties of this new single-parameter version of the utility function remain the same.

\section{Bi-criterion optimization}

In practice, bi-objective problems are often reduced to a single-objective problem by following one of two possible strategies:

(a) Modelling an objective function which is a weighted sum of the individual functions.

(b) Setting one objective, whose value is limited, as a constraint and then optimizing the other objective.

Moreover, the bi-objective problem

$$
\left\{\begin{array}{l}
\max \operatorname{Dmin}(X) \\
\max \operatorname{Util}(X) \\
\text { s.t. } \quad X \in \mathscr{S}
\end{array} \quad\right. \text { (DUMP) }
$$

can also be directly tackled by constructing the set of non-dominated (efficient or Pareto-optimal) points. The approach dealt with in this paper follows this methodology. For this purpose, two useful definitions are introduced.

Definition 1. A solution $X^{\prime}=\left(x^{\prime}, y^{\prime}\right)$ dominates the solution $X=(x, y)$ if and only if

$\operatorname{Dmin}\left(X^{\prime}\right) \geqslant \operatorname{Dmin}(X)$,

$\operatorname{Util}\left(X^{\prime}\right) \geqslant \operatorname{Util}(X)$.

Dominance is strict when at least one inequality is strict. 
Definition 2. $X$ is an efficient solution for the bi-objective problem if and only if no other solution $X^{\prime}$ exists which strictly dominates $X$.

Let $\mathscr{E}_{\mathscr{S}}$ be the set of those edges in the considered region $\Omega$ which are outside all forbidden zones $\mathscr{Z}_{k}$ (i.e., $\mathscr{E}_{\mathscr{S}}=\mathscr{E} \cap \mathscr{S}$ ) and let $\mathscr{N}_{\mathscr{S}}$ be the node set of the zone Voronoi diagram inside the feasible region $\mathscr{S}$.

Proposition 2. $\mathscr{E} \mathscr{S}$ is a dominating set of solutions for DUMP in $\mathscr{S}$.

Proof. Rectangular region $\Omega$ can be vertically scanned by means of segments of equation $x=\lambda$, with $0 \leqslant \lambda \leqslant M$, between $y=0$ and $y=L$. Each edge $e \in \mathscr{E} \mathscr{S}$ separates Voronoi regions inside $\mathscr{S}$.

Let $Q_{i}\left(\lambda, y_{i}(\lambda)\right)$ be the intersection points of vertical line $x=\lambda$ and $\mathscr{E} \mathscr{S}$, ordered by $y$-coordinates. These points constitute a finite dominating set for all points of vertical section $x=\lambda$ inside $\mathscr{S}$ :

Consider two consecutive points $Q_{i}$ and $Q_{i+1}$ which are in the contour of Voronoi region $Z_{k}(i)$. Let $Q(\lambda, y)$ be a generic point interior to Voronoi region $Z_{k}(i)$ inside vertical segment $\overline{Q_{i} Q_{i+1}}$ (Fig. 7). Due to the convexity property of the Euclidean distance, the maximum of function $d\left(Q, Z_{k}(i)\right)$ is reached at any extreme of segment $\overline{Q_{i} Q_{i+1}}$. Therefore, at least one of these relation pairs holds for all points $Q$ interior to segment $\overline{Q_{i} Q_{i+1}}$

$$
\left\{\begin{array} { l } 
{ \operatorname { U t i l } ( Q ) = \operatorname { U t i l } ( Q _ { i } ) , } \\
{ d ( Q , Z _ { k } ( i ) ) < d ( Q _ { i } , Z _ { k } ( i ) ) , }
\end{array} \left\{\begin{array}{l}
\operatorname{Util}(Q)=\operatorname{Util}\left(Q_{i+1}\right), \\
d\left(Q, Z_{k}(i)\right)<d\left(Q_{i+1}, Z_{k}(i)\right) .
\end{array}\right.\right.
$$

Proposition is a consequence of this fact.

Proposition 2 suggests to investigate the reduction of the number of non-efficient points inside set $\mathscr{E} \mathscr{g}$.

- Since each edge of the zone Voronoi diagram is either a straight line, a parabola or a hyperbola, a formal description is possible for each edge $e$ in terms of variable $y$, which acts as a parameter by taking values in a certain interval. Subsequently, each edge $e$ can be analytically expressed as follows:

$$
x=f_{e}(y), \quad \forall y \in\left[y_{1}(e), y_{2}(e)\right] .
$$

- This expression permits us to analyse utility function Util $(x(y))$ along each edge with respect to variable $y$ which takes values in its definition interval (i.e., $\forall y \in\left[y_{1}(e), y_{2}(e)\right]$ ). The interior of the intervals where the variation of the utility is monotonous can be explored by means of the cancellation of the factors which appear when the derivative of function Util $(x(y))$ is calculated:

$$
\frac{\mathrm{d}}{\mathrm{d} y} \operatorname{Util}(x(y))=x^{\alpha-1} \mathrm{e}^{-\beta x} x^{\prime}(y)(\alpha-\beta x) .
$$

In order to complete the analysis, the extremes of interval $\left[y_{1}(e), y_{2}(e)\right]$ are added for consideration. Let $\mathscr{N}_{\mathscr{B}}$ be the set of intersection points between edges $\mathscr{E}$ and boundaries $(x=0, x=M, y=0$ and $y=L)$ of the feasible region $\Omega$.

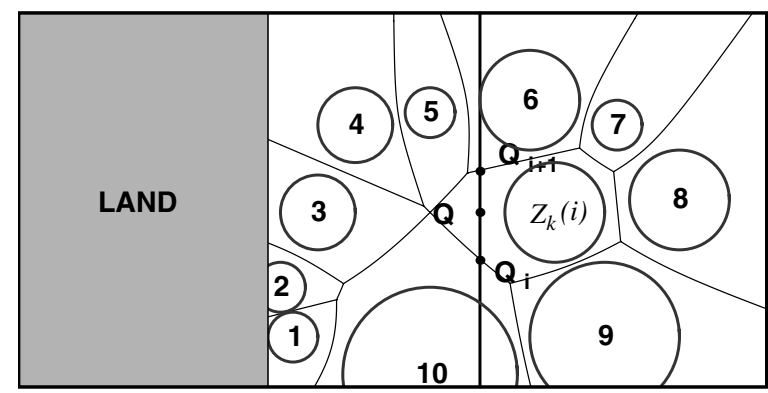

Fig. 7. Dominance along vertical segment $x=\lambda$. 
Fact 1: Solutions of equation $\frac{\mathrm{d}}{\mathrm{d} y} \mathrm{Util}(x(y))=0$ must satisfy one of these conditions:

- $x=0$ : the utility is at a minimum due to its definition.

- $x=\frac{\alpha}{\beta}$ : the utility is maximum as discussed in the above section. Let $\mathscr{N}_{\mathscr{U}}$ be the set of intersection points of the zone Voronoi diagram and the vertical line $x=\frac{\alpha}{\beta}$.

- $x^{\prime}(y)=0$ : solutions of this equation represent the most left-hand or the most right-hand points of the edge $e$ under consideration. Since the possible edges are either straight lines, parabolas or hyperbolas, the contribution of this term is reduced to extreme values $\left\{y_{1}(e), y_{2}(e)\right\}$ of the interval which describe the edge, and one additional point at most (only for the parabola and hyperbola cases) which is the single solution of the equation $x^{\prime}(y)=0$. Let $\mathscr{N}_{\mathscr{T}}$ be the set of points where an edge of set $\mathscr{E}$ has a vertical tangent line. If the edge involved were already a vertical segment then the inclusion of its highest and lowest points in set $\mathscr{N}_{\mathscr{T}}$ would be sufficient.

Fact 2: Since function $\operatorname{Dmin}(x(y), y)$ along each edge $e$ is convex, its minimum is reached either at one extreme point or at the intersection point between edge $e$ and the line which connects both centres of forbidden zones associated to edge $e$. Let $\mathscr{N}_{\mathscr{M}}$ be the set of those points where function $\operatorname{Dmin}(x(y), y)$ reaches its minimum in an edge of set $\mathscr{E}$.

Facts 1 and 2 lead to the deduction of a set of key points in order to later identify the non-dominated solutions for DUMP. To illustrate the methodology, Tuy's example has been used to find a solution for this biobjective problem (where $\alpha=4.5$ and $\beta=1$ ), and as considered in that paper, the solution space has been limited to the convex hull of forbidden zone centres (see Fig. 8) in order to enable an easier comparison. In this instance,

- Vertices labelled 1-9 are zone Voronoi diagram vertices inside the convex hull (set $\mathscr{N}_{\mathscr{S}}$ ).

- Vertices labelled 10-19 are points obtained by intersecting zone Voronoi diagram edges with the boundary of the aforementioned convex hull (set $\mathscr{N}_{\mathscr{B}}$ ).

- Vertices labelled 20-21 belong to vertical line $x=\frac{\alpha}{\beta}$ and an edge of the Voronoi zone diagram (set $\mathscr{N}_{\mathscr{U}}$ ).

- Finally, vertex 22 is a solution of the algebraic equation $x^{\prime}(y)=0$ (set $\mathscr{N}_{\mathscr{T}}$ ).

- For simplicity, points of set $\mathscr{N}_{\mathscr{M}}$ have not been taken into account in the example since no point, where function $\operatorname{Dmin}(x(y), y)$ is maximized along edges in $\mathscr{E}$, is efficient.

Note that set $\mathscr{N}_{\mathscr{K}}=\mathscr{N}_{\mathscr{S}} \cup \mathscr{N}_{\mathscr{B}} \cup \mathscr{N}_{\mathscr{U}} \cup \mathscr{N}_{\mathscr{T}} \cup \mathscr{N}_{\mathscr{M}}$ of key points collects all local optima for some single objective. Each point $Q_{n} \in \mathscr{N}_{\mathscr{K}}, \forall n=1, \ldots,\left|\mathscr{N}_{\mathscr{K}}\right|$, generates a point $\left(u_{n}, d_{n}\right)=\left(\operatorname{Util}\left(Q_{n}\right), \operatorname{Dmin}\left(Q_{n}\right)\right)$ on the plane of values of objective functions. Our task consists of providing the shortest list of non-inferior (nondominated or efficient) points.

The methodology called NISE, non-inferior set estimation (Cohon, 1978), is an appropriate tool for the identification of extreme non-dominated solutions from the existing set of local optimum points (Fig. 9). The NISE algorithm uses a weighting method to generate an approximation of the non-inferior solution set. This technique is iterative and provides a quick convergence at the solutions which lie on the convex hull of the non-inferior solution set.

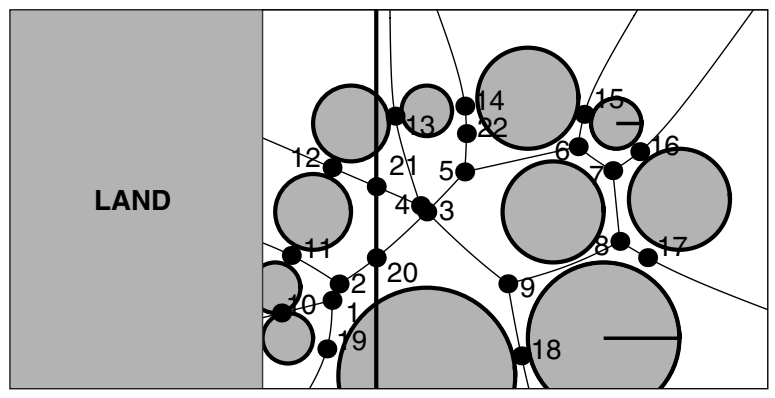

Fig. 8. Key vertices for $\alpha=4.5$ and $\beta=1$. 


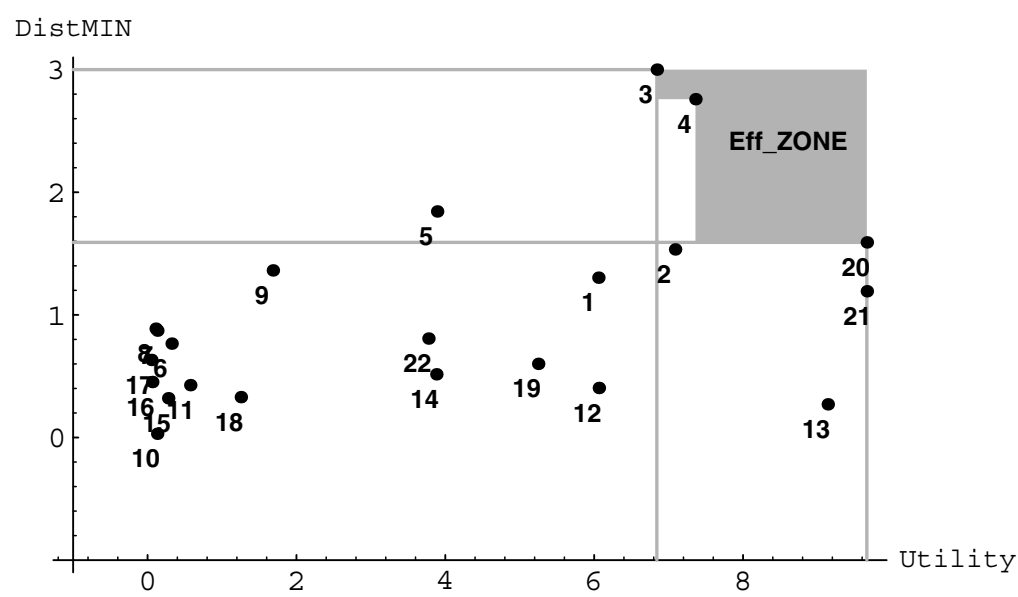

Fig. 9. Efficiency of key points for $\alpha=4.5$ and $\beta=1$.

We propose the following algorithm, composed by one main program and one recursive subroutine, which uses the NISE method to find an approximation to the set of efficient solutions Eff $\subset \mathscr{N}_{\mathscr{K}}$. The termination criterion combines a maximum depth of recursion (Maxlevel $\in \mathbb{N}$ ) and a maximum predetermined error $(\epsilon>0)$ to control the accuracy (step 1).

The main program identifies endpoints $A$ and $B$ of $\mathscr{N}_{\mathscr{K}}$ by maximizing the single objectives separately (steps 3 and 4). These points provide the initial arguments for the subroutine called from the main program (step 6).

\section{main program}

1. Fix Maxlevel $>0$ and $\epsilon>0$;

2. Eff $\varnothing ;$

3. Obtain $u_{\max }:=\max _{Q_{n} \in \mathcal{N}_{\mathscr{X}}} \operatorname{Util}\left(Q_{n}\right)$. Let $n_{u}$ be the value of index where this maximum is reached. In the case of a tie, consider the point where the second objective is higher. Let $A:=\left(\operatorname{Util}\left(Q_{n_{u}}\right), \operatorname{Dmin}\left(Q_{n_{u}}\right)\right)$.

4. Obtain $d_{\text {max }}:=\max _{Q_{n} \in \mathcal{N}_{\mathscr{K}}} \operatorname{Dmin}\left(Q_{n}\right)$. Let $n_{d}$ be the value of index where this maximum is reached. In the case of a tie consider the point where the first objective is higher. Let $B:=\left(\operatorname{Util}\left(Q_{n_{d}}\right), \operatorname{Dmin}\left(Q_{n_{d}}\right)\right)$.

5. Eff $\quad$ Eff $\cup\{A, B\}$;

6. Iterate $[1, \epsilon, A, B]$.

In the subroutine the coordinates of points $A$ and $B$ provide a trade-off of both objectives based on line segment $A B$ :

$$
\left(b_{2}-a_{2}\right)\left(x-a_{1}\right)+\left(a_{1}-b_{1}\right)\left(y-a_{2}\right)=0 .
$$

By maximizing the objective induced by the function $\left(b_{2}-a_{2}\right)\left(\mathrm{Util}-a_{1}\right)+\left(a_{1}-b_{1}\right)\left(\right.$ Dmin $\left.-a_{2}\right)$ inside $\mathscr{N}_{\mathscr{K}}$ (step 1.1), an extreme non-inferior solution $H$ can be obtained. If this new solution represents a sensitive improvement (condition 1.2.2) then is added to set Eff and the subroutine invokes itself to continue with the exploration of $\mathscr{N}_{\mathscr{K}}$.

subroutine Iterate [level, $\left.\epsilon, A\left(a_{1}, a_{2}\right), B\left(b_{1}, b_{2}\right)\right]$

1 . If level $\leqslant$ Maxlevel then

\subsection{Obtain}

$$
\max _{Q_{n} \in \mathscr{N}_{\mathscr{K}}}\left(b_{2}-a_{2}\right) \cdot\left(\operatorname{Util}\left(Q_{n}\right)-a_{1}\right)+\left(a_{1}-b_{1}\right) \cdot\left(\operatorname{Dmin}\left(Q_{n}\right)-a_{2}\right)
$$

1.2. If the maximum found is positive then 
1.2.1. Let $n_{c}$ be the value of index where this maximum is reached. In the case of a tie consider the first point obtained. Let $H:=\left(\mathrm{Util}\left(Q_{n_{c}}\right), \operatorname{Dmin}\left(Q_{n_{c}}\right)\right)$.

1.2.2. If distance from $H$ to line $\mathrm{AB}$ is higher than $\epsilon$ then

Eff $\leftarrow$ Eff $\cup\{H\}$

Iterate $[$ level $+1, \epsilon, A, H]$;

Iterate $[$ level $+1, \epsilon, H, B]$

else Return.

\section{else Return.}

\section{else Return.}

In the instance based on Tuy's example, the application of a previous algorithm asserts that key points 3,4 and 20 are non-dominated. Since, in this example, the aforementioned points are adjacent nodes in the neighbourhood graph associated to the zone Voronoi diagram, the remainder interior points included in the edge segments, which directly connect these extreme points, must be additionally considered as possible non-dominated locations. In fact, the interior points of edges $(3,4)$ and $(3,20)$ are efficient, as is shown in Fig. 10, where the values for the considered objectives have been calculated for each interior point and represented as arcs of continuous curves in the plane. Additional efficient points could be obtained along edges $(4,13)$ and $(4,21)$, taking into account that key point 4 is interior to the efficiency zone (see Fig. 9); nevertheless, this potential efficiency is not acquired at any point in this case (Fig. 10). Fig. 11 shows the arcs containing the optimal locations for the endpoint of a waste pipeline for this example.

Moreover, it should be noted that the set of non-inferior locations for the bi-criterion problem can also contain isolated vertices. This occurs when a non-inferior solution has no next-edge neighbour which shares

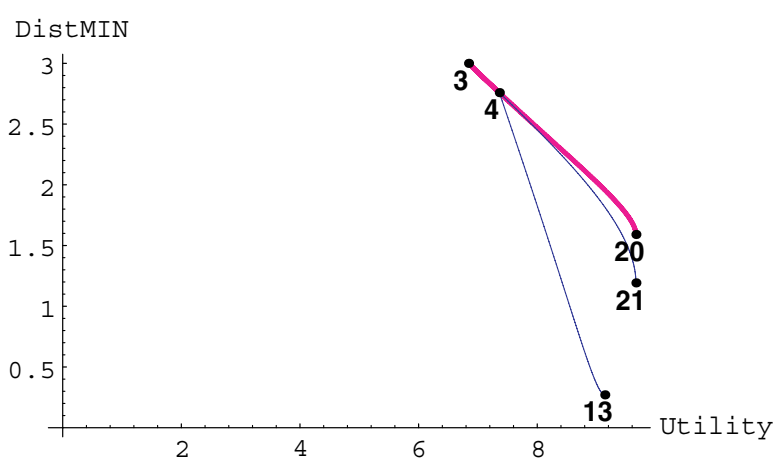

Fig. 10. Efficiency of edge segments.

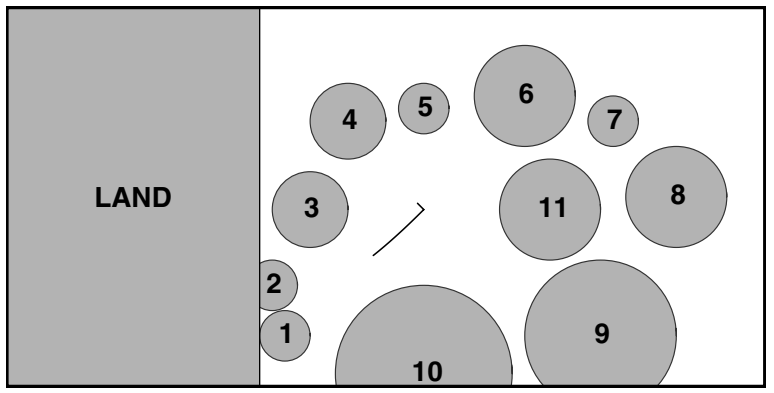

Fig. 11. Optimal locations for the pipeline endpoint. 


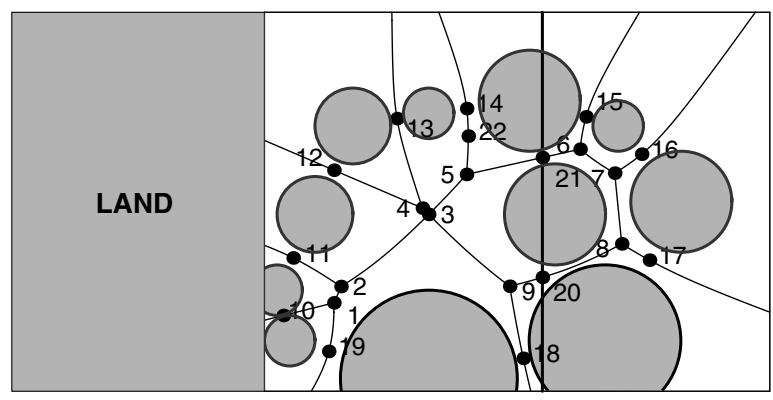

Fig. 12. Key vertices for $\alpha=11$ and $\beta=1$.

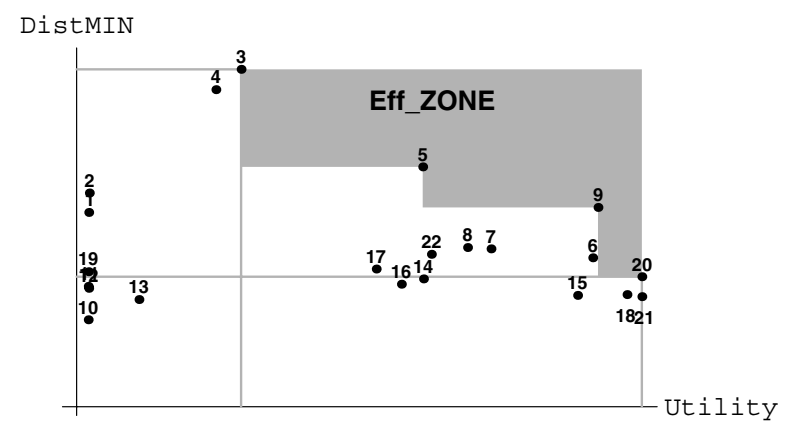

Fig. 13. Efficiency of key points for $\alpha=11$ and $\beta=1$.

the quality of being efficient. Attention may be drawn to the sensitivity of the solution set with respect to the utility function. Fig. 12, obtained for a new value of parameter $\alpha=11$ (whilst maintaining the value of $\beta$ ), shows this sensitivity, which leads to changes in the set of efficient key points, as is shown in Fig. 13.

Efficiency of key points is shown in Fig. 13. Consequently, the edges to explore, since these can contain potential efficient points, are $(3,5),(3,9),(9,20),(9,18),(5,21)$ and $(5,22)$.

\section{Conclusions and extensions}

In this paper we have presented a methodology for finding the efficient set of points where the endpoint (diffuser) of a waste pipeline must be located in order to minimize the negative impact on marine environment. The region under study has been assumed to be a rectangle which includes zones of biological interest, geometrically modelled as rectangles and circles.

The length of the pipe has been associated to a utility function described by a generalized gamma function. Since intensity of pollution has been assumed to be inversely proportional to the Euclidean distance, the location problem was formulated as a bi-criterion problem in which both the minimum distance to protected zones and the utility function had to be maximized. An efficient set of solutions has been identified along edges of the zone Voronoi diagram by means of an approach based upon the NISE method.

Building the zone Voronoi diagram is feasible since its computational complexity is equivalent to the complexity required to generate the classic Voronoi diagram (Okabe et al., 2000). The finite dominating set of points used to describe the solution contains, in the worst case, $6\left|\mathscr{E}_{\mathscr{S}}\right|$, where $\left|\mathscr{E}_{\mathscr{S}}\right|$ represents the number of edges of the zone Voronoi diagram inside the considered region $\mathscr{S}$. Coefficient 6 is included since all the following points need to be considered for each edge: two extreme points, two additional points (in the worst case) obtained by intersecting the edge with vertical line $x=\frac{\alpha}{\beta}$, one more point if at this point the line tangent to the curve associated to the edge is vertical and, finally, one further point in the edge where distance to adjacent zones is minimal (this key point is usually a non-efficient point). 
The structure of the solution set consists of isolated vertices and continuous arcs of edges in the zone Voronoi diagram. This fact suggests the possibility of a further selection from the solution set by including additional objectives which could be taken into account later.

Instead of the Euclidean distance considered in the context, polyhedral gauges may be more adequate for the modelling of distances in the presence of prevailing undercurrents. Other approaches to a distance concept based on oxygen level and/or the need for oxygen in polluted waters could improve the suitability of the model (see Haider et al., 2003). This extension would require the previous resolution of the corresponding ordinary differential equations of first order and/or partial differential equations to determine the iso-affectation curves.

\section{Acknowledgements}

This work has been supported in part by a grant from the Spanish Research Projects BFM2000-1052-C0201 and BFM2003-04062. This paper is a major extension of the work presented at the XIV EWGLA Meeting2003, held in Corfu (Greece). Authors are grateful to anonymous referees for their valuable suggestions and comments.

\section{Appendix. Proof of Proposition 1}

The boundaries of the region under study and the contours of the protected zones are labelled in order to carry out a full analysis of cases:
B1. Axis $O Y$.
B2. Axis $O X$.
B3. Vertical delimitation $x=M$.
B4. Horizontal delimitation $y=L$.
C1. Contour of circle $C(A, r)$
C2. Contour of rectangle $R(V, l, h)$.

1. The bisector of pair [B1, B2] is straight line $y=x$.

2. The bisector of pair [B1, B3] is vertical line $x=M / 2$.

3 . The bisector of pair $[\mathrm{B} 1, \mathrm{~B} 4]$ is straight line $y=-x+L$.

4. The bisector of pair $[\mathrm{B} 1, \mathrm{C} 1]$ is a parabola (see Fig. 14).

By expressing the property $d_{2}(X, A)-r=d_{2}(X$, axis $O Y)$ in terms of Cartesian coordinates, the equation

$$
\left(x-a_{1}\right)^{2}+\left(y-a_{2}\right)^{2}=(x+r)^{2}
$$

is obtained. It represents the parabola

$$
x-\frac{a_{1}-r}{2}=\frac{1}{2\left(r+a_{1}\right)}\left(y-a_{2}\right)^{2}, \quad \text { for } y \in[0, L]
$$

with horizontal axis, whose vertex is at $Q\left(\frac{a_{1}-r}{2}, a_{2}\right)$ and whose focus point is at $F\left(\frac{a_{1}-r}{2}+\frac{1}{8\left(r+a_{1}\right)}, a_{2}\right)$.

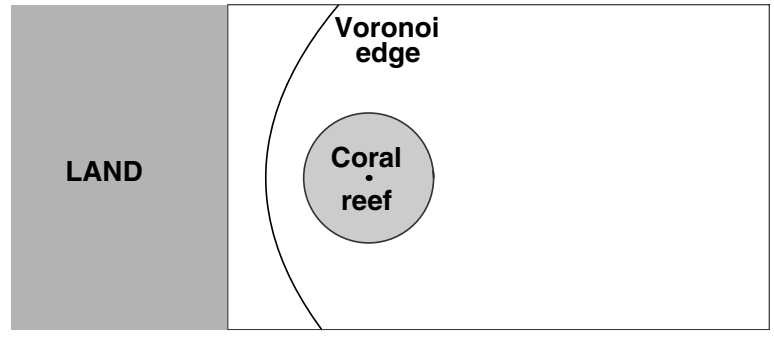

Fig. 14. The bisector of pair [axis $x=0$, circle $C$ ]. 


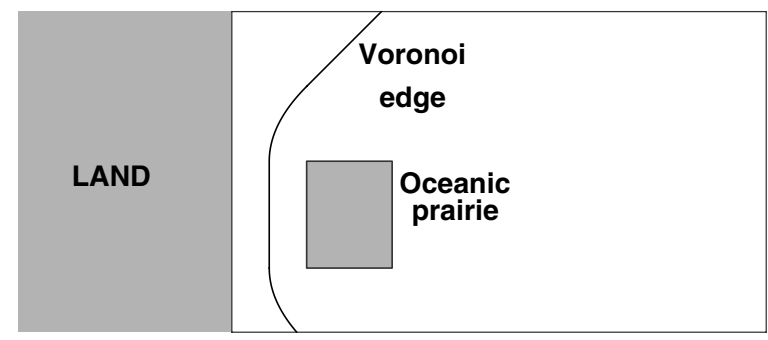

Fig. 15. The bisector of pair [axis $x=0$, rectangle $R$ ].

5. The bisector of pair [B1, C2] is a piece-wise curve composed of an alternating sequence of parabola arcs and straight segments (see Fig. 15).

- The central part of the bisector curve, vertical segment $x=v_{1} / 2$, is the corresponding to the pair composed of axis $x=0$ and rectangle side $x=v_{1}$.

- Since the condition $d_{2}(X$, point $)=d_{2}(X$, straight line $)$ yields a parabola, the higher continuation of the bisector curve is a parabola arc where its focal point is rectangle vertex $\left(v_{1}, v_{2}+h\right)$ whereas the parabola vertex is $\left(\frac{v_{1}}{2}, v_{2}+h\right)$. This parabola is given by

$x=\frac{v_{1}}{2}+\frac{1}{2 v_{1}}\left(y-v_{2}-h\right)^{2} ; \quad \forall y \in\left[v_{2}+h, v_{2}+h+v_{1}\right]$.

- The bisector higher extension continues with the segment $y=x+v_{2}+h$, for $x \in\left[v_{1}, v_{1}+l\right]$, obtained by imposing the condition $\left.d_{2}(X \text {, straight line })_{1}\right)=d_{2}\left(X\right.$, straight line $\left.e_{2}\right)$ for axis $x=0$ and upper side $y=v_{2}+h$ of rectangle.

- The end of upper part of the bisector curve is the arc of parabola whose focal point is at $\left(v_{1}+l, v_{2}+h\right)$ : $x=\frac{v_{1}+l}{2}+\frac{l}{2\left(v_{1}+l\right)}\left(y-\left(v_{2}+h\right)\right)^{2} ; \quad \forall y \in\left[v_{2}+v_{1}+l+h, L\right]$.

For the deduction of the lower extreme of the bisector curve, the reasoning is similar. The lower extension of the central part consists of

- The arc of parabola with focal point at $\left(v_{1}, v_{2}\right)$ and vertex at $\left(\frac{v_{1}}{2}, v_{2}\right): x=\frac{v_{1}}{2}+\frac{1}{2 v_{1}}\left(v_{2}-y\right)^{2} ; \forall y \in\left[v_{2}-\right.$ $\left.v_{1}, v_{2}\right]$.

- The segment $y=v_{2}-x ; \forall x \in\left[v_{1}, v_{1}+l\right]$.

- The arc of parabola with focal point at $\left(v_{1}+l, v_{2}\right)$ :

$$
x=\frac{v_{1}+l}{2}+\frac{l}{2\left(v_{1}+l\right)}\left(y-v_{2}\right)^{2} ; \quad \forall y \in\left[0, v_{2}-v_{1}-l\right] .
$$

6. Similar developments can be applied to obtain all bisector curves where axis $y=0$, boundary $y=L$ and boundary $x=M$ are involved.

7. The bisector curve for the pair of circles $C_{1}\left(A_{1}, r_{1}\right)$ and $C_{2}\left(A_{2}, r_{2}\right)$ generates one branch of hyperbola curve $d_{2}\left(X, A_{1}\right)-d_{2}\left(X, A_{2}\right)=r_{1}-r_{2}$.

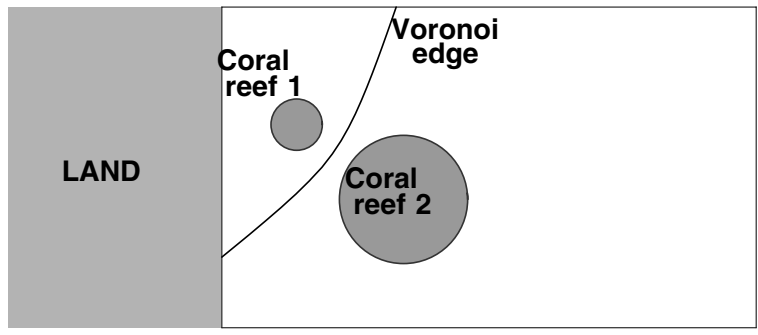

Fig. 16. The bisector of pair [circle $C_{1}$, circle $C_{2}$ ]. 


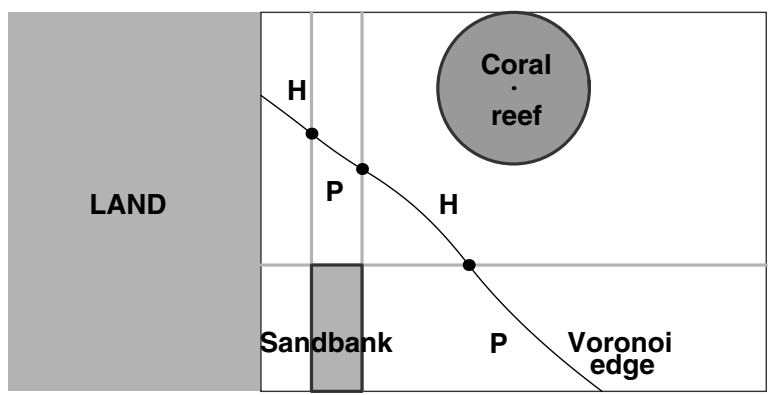

Fig. 17. The bisector of pair [circle $C$, rectangle $R$ ].

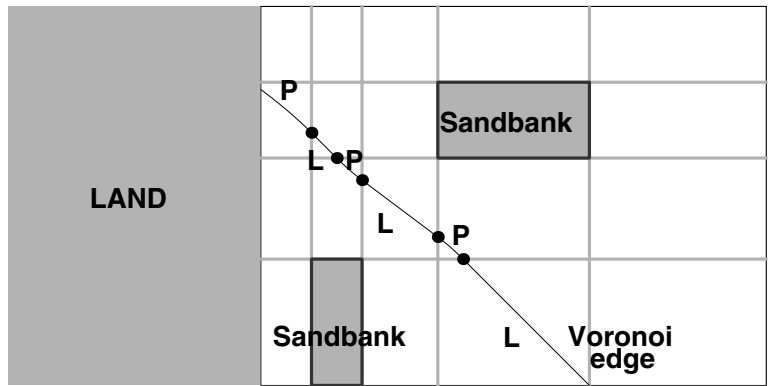

Fig. 18. The bisector of pair [rectangle $R_{1}$, rectangle $R_{2}$ ].

If $r_{2}>r_{1}$ then $d_{2}\left(X, A_{2}\right)>d_{2}\left(X, A_{1}\right)$ and hence the hyperbola branch turns around the circle with the smallest radius $r_{1}$ (see Fig. 16).

8. The bisector curve (see Fig. 17) of the pair composed of circle $C(A, r)$ and rectangle $R(V, l, h)$ is a continuous union of arcs of hyperbola branches $(\mathrm{H})$ and parabolas arcs $(\mathrm{P})$ since

- The bisector curve for point $V$ (a rectangle vertex) and the circle is expressed as $d_{2}(X, A)-r=d_{2}(X, V)$; it implies equation $d_{2}(X, A)-d_{2}(X, V)=r$ which identifies the branch of the hyperbola whose focal points are at points $A$ and $V$, and whose distance between vertices is $r$.

- The bisector curve for a vertical (or horizontal) edge of rectangle $R(V, l, h)$ and the circle is an arc of a parabola.

9. Finally, the bisector curve (see Fig. 18) corresponding to rectangle pairs $R_{1}\left(V_{1}, l_{1}, h_{2}\right)$ and $R_{2}\left(V_{2}, l_{2}, h_{2}\right)$ is a continuous union of portions of mediatrix lines $(\mathrm{L})$ and parabolas $(\mathrm{P})$.

\section{References}

Calzadilla Pérez, A., Damen, M.C.J., Geneletti, D., Hobma, T.W., 2002. Monitoring a recent delta formation in a tropical coastal wetland using remote sensing and GIS. case study: Guapo river delta, Laguna de Tacarigua, Venezuela. Environment, Development and Sustainability 4, 201-219.

Caerio, S., Painho, M., Goovaerts, P., Costa, H., Sousa, S., 2003. Spatial sampling design for sediment quality assessment in estuaries. Environmental Modelling \& Software 18, 853-859.

Cohon, J.L., 1978. Multiobjective Programming and Planning. Academic Press, New York.

EU Directive 1992. Council Directive 92/43/EEC of 21 May 1992 on the conservation of natural habitats and wild fauna and flora. Official Journal of the European Communities 35(L 206), 7-489.

Fernandez, F., Puerto, J., Rodriguez-Chía, A.M., 1997. A maxmin location problem with non-convex feasible region. Journal of Operational Research Society 48, 479-489.

Haider, S., Paquier, A., Morel, R., Champagne, J.Y., 2003. Urban flood modelling using computational fluid dynamics. Water \& Maritime Engineering 156, 129-135. 
He, C., 2003. Integration of geographic information systems and simulation model for watershed management. Environmental, Modelling \& Software 18, 809-813.

Instituto Nacional de Estadística, 2004. Censo de Población y Viviendas 2001. Report in Spanish available at electronic address: $<$ http:// www.ine.es/infoine>.

Jayatissa, L.P., Guero, M-C., Hettiarachchi, S., Koedam, N., 2002. Changes in vegetation cover and socio-economic transitions in a coastal lagoon (Kalametiya, Sri Lanka), as observed by teledetection and ground truthing, can be attributed to an upstream irrigation scheme. Environment, Development and Sustainability 4, 167-183.

Marbá, N., Duarte, C.M., Cebrián, J., Gallegos, M.E., Olesen, B., Sand-Jensen, K., 1996. Growth and population dynamics of posidonia oceanica on the Spanish Mediterranean coast: Elucidating seagrass decline. Marine Ecology Progress Series 137, $203-213$.

Melachrinoudis, E., Cullinane, T.P., 1985. Locating an undesirable facility within a geographical region using the maxmin criterion. Journal of Regional Science 25, 115-127.

Melloul, A.J., Collin, M.L., 2002. Prioritization of sustainable groundwater management needs: The case of the Israel's stressed Coastal Aquifer. Environment, Development and Sustainability 4, 347-360.

Okabe, A., Boots, B., Sugihara, K., Chiu, S.N., 2000. Spatial Tessellations: Concepts and Applications of Voronoi Diagrams. Wiley, Chichester.

Ortúzar, J.D., Willumsen, L.G., 2001. Modelling Transport, third ed. Wiley, Chichester.

Qin, Z., Xu, Y., Zhu, B., 2003. On some optimization problems in obnoxious facility location. In: Lecture Notes in Computer Science, Computing and Combinatorics: 6th Annual International Conference, COCOON 2000 (Sydney, Australia), vol. 1858/2000. SpringerVerlag, Heidelberg, pp. 320-329.

Reiff, F.M. 2002. Small diameter HDPE (high-density polyethylene) submarine outfalls. Technical Report OPS/CEPIS/PUB/00.60. Pan American Center for Sanitary Engineering and Environmental Sciences (CEPIS). Washington, DC.

Rockafellar, R.T., 1970. Convex Analysis. Princeton University Press, New Jersey.

Tuy, H., Nghia, N.D., Vinh, L.S., 2003. A discrete location problem. Acta Mathematica Vietnamica 28, $185-199$.

Tuy, H., Minoux, M., Hoai-Phuong, N.T., in press. Discrete monotonic optimization with application to a discrete location problem. SIAM Journal on Optimization.

Worldwide Fund for Nature/Adena 2000. Las Praderas de Posidonia: Importancia y Conservación. Report in Spanish available at electronic address $<$ http://www.wwf.es $>$. 